\title{
INFORMÁTICA NA ESCOLA: ESTUDO DE CASO PARA AS ESCOLAS PÚBLICAS ESTADUAIS DE VIDEIRA/SC
}

http://dx.doi.org/10.5902/2318133831111

\author{
Leila Lisiane Rossi \\ Jean Carlo Corso \\ Gilson Ribeiro Nachtigall \\ Angela Maria Crotti da Rosa \\ Instituto Federal Catarinense, campus Videira, Brasil. \\ Luiz Gustavo Moro Senko \\ Instituto Federal Catarinense, campus Brusque, Brasil.
}

\begin{abstract}
Resumo
No presente artigo mostram-se os resultados de um projeto de pesquisa cujo principal objetivo foi o desenvolvimento de uma ferramenta web para auxiliar na análise dos dados relacionados ao uso e a necessidade de tecnologias no ensino nas escolas públicas estaduais urbanas de Videira/SC. Em um projeto anterior foi desenvolvida uma ferramenta similar, porém, voltada às escolas municipais de Videira/SC. Inicialmente foram aplicados questionários dirigidos aos alunos e aos professores e, posteriormente, foi desenvolvida a ferramenta usando software livre com vistas a permitir a visualização dos dados de forma multidimensional, ou seja, várias dimensões e diferentes níveis de detalhamento para facilitar a análise dos dados na tomada de decisão.
\end{abstract}

Palavras-chave: software livre; tomada de decisão; tecnologia.

\section{Informatics in the school: case study in the public schools of Videira city}

\begin{abstract}
The present paper shows the results of a research project that describes the web tool to support the data analysis in the research project about the technological needs in the public schools from Videira city. In a previous project, a similar tool was developed for the municipal schools of Videira/SC. For some schools was developed a similar project. At first, questionnaires were applied to teachers and students and then, the tool was created using free software allows the multidimensional data visualization, which means, in other words, different dimensions and levels of details which makes it easier to analyze data in the decision making process.

Key-words: free software; decision making; technology.
\end{abstract}




\section{Introdução}

A $s$ tecnologias estão cada vez mais presentes em nossas vidas e nos tornamos cada vez mais dependentes delas. Praticamente em todas as áreas lé possível encontrar tecnologia de uma forma ou de outra, o que não é diferente na educação, seja na modalidade a distância ou presencial.

Os professores estão cada vez mais conscientes de que a tecnologia se tornou uma grande aliada nas atividades de ensino. Assim, com o objetivo de contribuir com as escolas públicas estaduais de Videira/SC, foi realizada uma pesquisa com professores e alunos para conhecer as tecnologias usadas nas aulas, bem como as dificuldades no manuseio dos equipamentos e o interesse em cursos gratuitos de informática.

Como principal resultado do projeto foi desenvolvida uma ferramenta web que permite analisar os dados de maneira amigável, facilitando a tomada de decisão em relação à adoção de políticas educacionais.

\section{Inclusão digital}

As gerações nascidas posteriormente à década de 1980 são consideradas nativos digitais pela facilidade de acesso e uso das tecnologias. Por outro lado, as demais gerações que, geralmente, possuem algum tipo de dificuldade e que, de uma forma ou de outra, procuram se adaptar a elas e aprendem a usá-las são os chamados migrantes digitais (Prensky, 2001).

Mesmo considerando essas facilidades dos chamados nativos digitais, sabe-se da situação de grande parte das escolas públicas em relação à dificuldade de acesso às tecnologias e da falta de apoio técnico. Diante deste cenário é importante a realização de parcerias com outras instituições de ensino por meio de projetos de extensão, pois acredita-se que o uso adequado de tecnologias nas escolas é relevante, tanto para os professores, ao contribuir para a capacitação profissional, quanto para os alunos, ao proporcionar a oportunidade de aprendizado de forma dinâmica e interativa.

Como forma de contribuir com a inclusão digital e dar sequência ao projeto anterior voltado para as escolas municipais (Rosa et. al., 2016), foi realizada uma pesquisa nas escolas públicas estaduais urbanas de Videira/SC. No total são oito escolas: Governador Lacerda, Ceja, Madre Terezinha Leoni, Professora Adelina Regis, Josefina Caldeira de Andrade, Padre Bruno Pokolm, Esther Crema Marmentini e Inspetor Eurico Rauem. O foco em escolas públicas deve-se ao fato de que, geralmente, estas são mais carentes de recursos tecnológicos.

\section{Informática na escola: estudo de caso}

A seguir são apresentados os trabalhos correlatos encontrados nas pesquisas realizadas. Uma das pesquisas mais similares foi o projeto desenvolvido anteriormente pela mesma instituição sobre o uso e a necessidade de tecnologias nas escolas municipais de Videira/SC (Rosa et.al., 2016). Acredita-se que existam pesquisas relacionadas, mas possivelmente não encontradas pela forma diferenciada de 
armazenamento, recuperação e apresentação dos dados. O presente trabalho foi desenvolvido no IFC-Videira como um projeto de pesquisa e teve como objetivo conhecer o perfil das escolas públicas estaduais de Videira/SC em relação ao uso e a necessidade do conhecimento de tecnologias.

Para o desenvolvimento da ferramenta foi escolhido software livre como o Pentaho BI Server (Pentaho, 2017), para o Data Webhouse, e a Weka (Weka, 2017) para a mineração de dados. Desenvolveu-se e aplicou-se, inicialmente, um questionário aprovado pelo Comitê de Ética, para a entrada de dados a partir das respostas obtidas nas escolas. Com base neste formulário as informações foram carregadas para a base de dados relacional (Elmasri; Navathe, 2011), pela ferramenta PostgreSQL (Milani, 2008).

Posteriormente o modelo Multidimensional Estrela (Kimball, 2002) foi escolhido para a criação do cubo, permitindo, assim, a disponibilização dos dados em uma tabela Fato centralizada, a qual contém as medidas necessárias para a realização das consultas Analíticas online - Olap - (Inmon, 1996) e várias tabelas Dimensão ligadas a ela. O cubo Professor possui várias tabelas Dimensão, sendo que aqui serão destacadas as principais delas: trabalha com último ano do ensino fundamental, usa computador ou similar nas aulas, dificuldades dos alunos ao usar computador, possui incentivo a capacitação, possui ajuda de profissional de tecnologia, faria curso de tecnologia gratuito, entre outras.

A tabela Fato Professor apresenta as medidas necessárias para a realização das consultas Olap, tais como a quantidade de professores que trabalham com o último ano fundamental, a quantidade de professores que possuem ajuda de profissional de informática, entre outras, ou seja, funções de agregação/medidas relacionadas às tabelas dimensão do cubo Professor. O cubo Aluno possui Dimensões: se o aluno usa computador ou similar nas aulas, se o aluno usa laboratório de informática na escola, se os professores usam tecnologias nas suas aulas, entre outras. A tabela Fato Aluno apresenta as medidas necessárias para a realização das consultas Olap, tais como a quantidade de alunos que usam computador ou similar, a quantidade de alunos que fariam um curso de tecnologia gratuito, outras, ou seja, funções de agregação/medidas relacionadas às tabelas dimensão do cubo Aluno.

A partir do modelo multidimensional armazenado no PostgreSQL (Postgresql, 2016), foi gerado o cubo com a Extensible Markup Language - XML - usando a ferramenta Schema Workbench (Pentaho, 2016). Pelo cubo XML é possível criar consultas Olap e visualizá-las de forma multidimensional na web com a ferramenta Pentaho BI Server (Pentaho, 2016). Estas ferramentas foram escolhidas por serem software livre, seguindo, portanto, as recomendações do governo federal. A consulta Olap é realizada com auxílio da linguagem de manipulação de dados Multidimensional Expressions - MDX. Trata-se de uma linguagem semelhante à Structured Query Language - SQL - (Silberschatz, 2006), porém, utilizada especificamente em Data Webhouse. A ferramenta de mineração de dados Weka (Weka, 2016), aplicada no cubo gerado, permitiu resultados de associação e clusterização considerados importantes para avaliar as preferências e necessidades das escolas.

A figura 1, a seguir, apresenta como o professor poderia usar o computador nas aulas na opinião dos alunos. É apresentado o total por atividade sugerida considerando todas as escolas. 
Figura 1 -

Como o professor poderia usar o computador nas aulas.

\begin{tabular}{|l|r|}
\hline & Measures \\
\hline Como_professor_poderia_usar_computador_aulas & total \\
\hline All como_professor_poderia_usar_computador_aulass & 158 \\
\hline Jogos_Educativos & 21 \\
\hline Jogos_Educativos_e_Pesquisas_Internet & 4 \\
\hline Jogos_Educativos_Videos & 3 \\
\hline Jogos_Educativos_Videos_e_Pesquisas_Internet & 5 \\
\hline Jogos_Videos_Pesquisas_Internet_e_Outras_Formas & 4 \\
\hline Outras_Formas & 8 \\
\hline Pesquisas_Internet & 58 \\
\hline Pesquisas_Internet_e_Outras_Formas & 3 \\
\hline Videos & 30 \\
\hline Videos_e_Outras_Formas & 1 \\
\hline Videos_e_pesquisas_na_internet & 19 \\
\hline Videos_e_pesquisas_na_internet_e_Outras_Formas & 2 \\
\hline
\end{tabular}

Por uma consulta Olap, como no caso da figura 1, se escolher por escola é possível visualizar os dados por níveis de detalhamento - Drill-Down-Up -, ou seja, o usuário pode escolher, por exemplo, a resposta por escola separadamente.

\section{Resultados obtidos}

O principal resultado foi a criação de uma ferramenta web pela qual é possível conhecer e analisar facilmente o uso de tecnologias e as preferências dos alunos e professores das escolas pesquisadas. A figura 2 mostra que a maioria dos alunos considera necessário o uso de tecnologias nas aulas. 
Figura 2 -

Consulta Olap: considera necessário computador nas atividades escolares.

\section{Measures}

\section{total}

\section{acha_necessario_computador_atividades_escolares}

- - All

Nao $\bullet$ Sim

acha_necessario_computador_atividades_escolaress

A figura 3 apresenta o resultado sobre a preferência por aulas com o uso de tecnologias. Foram aplicadas algumas técnicas de mineração de dados (Witten; Frank, 2005) como as regra de associação - algoritmo Tertius usando a ferramenta Weka (Weka, 2016) e outras continuam sendo testadas. Pelas regras de associação geradas é possível identificar, por exemplo, que os alunos que tem preferência por aulas com tecnologia também tem interesse em fazer um curso de informática gratuito.

Figura 3 -

Consulta Olap: preferência por aulas com tecnologias.

\begin{tabular}{|c|c|c|c|}
\hline & & & Measures \\
\hline prefere_aulas_com_uso_tecnologias & professores_usam_tecnologias_nas_aulas & usa_lab_info_escola & - total \\
\hline GAll prefere_aulas_com_uso_tecnologiass & ${ }^{\dagger}$ All professores_usam_tecnologias_nas_aula & ${ }^{+}$All usa_lab_info_escolas & 158 \\
\hline NaO & ${ }^{\dagger}$ All professores_usam_tecnologias_nas_aula & $\Psi^{+}$All usa_lab_info_escolas & 9 \\
\hline Sim & ${ }^{\dagger}$ All professores_usam_tecnologias_nas_aula & ${ }^{+}$All uso_ab_info_escolas & 149 \\
\hline
\end{tabular}

A figura 4 apresenta a consulta Olap sobre os computadores com o objetivo de identificar se eles são adequados na escola. É possível verificar que a maioria dos professores consideram que não são adequados para as suas aulas. 
Figura 4 -

Consulta Olap: computadores adequados na escola.

\begin{tabular}{|c|c|c|c|}
\hline \multicolumn{4}{|l|}{ Measures } \\
\hline \multicolumn{4}{|l|}{ total } \\
\hline \multicolumn{4}{|l|}{ computadores_escola_adequados } \\
\hline $\begin{array}{l}\text { - All } \\
\text { computadores_escola_adequadoss }\end{array}$ & Nao & Sem_Resposta & Sim \\
\hline escola & escola & escola & escola \\
\hline - + All escolas & - + All escolas & - + All escolas & - ${ }^{+}$All escolas \\
\hline 54 & 30 & 2 & 22 \\
\hline
\end{tabular}

A figura 5 apresenta um exemplo de consulta analítica pela qual é possível identificar que a maioria dos professores fariam um curso de tecnologia gratuito.

Figura 5 -

Consulta Olap: faria curso de tecnologia gratuito.

\begin{tabular}{|c|c|c|c|c|c|c|}
\hline \multirow[b]{4}{*}{ escola } & \multicolumn{6}{|l|}{ Measures } \\
\hline & \multicolumn{6}{|l|}{ total } \\
\hline & \multicolumn{6}{|l|}{ faria_curso_tecnologia_gratuito } \\
\hline & $\begin{array}{l}\text { - All } \\
\text { faria_curso_tecnologia_gratuito }\end{array}$ & & Nao & - Sem_Resposta & $\bullet$ & Sim \\
\hline All escolas & 5 & & 5 & 3 & 3 & 46 \\
\hline CEJA & 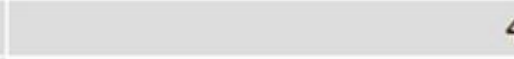 & 4 & & & & 4 \\
\hline Esther Crema Marmentini & ( & 6 & 1 & 1 & 1 & 4 \\
\hline Governador Lacerda & 5 & 5 & 2 & 1 & 1 & 2 \\
\hline Inspetor Eurico Rauem & 10 & & 1 & & & 9 \\
\hline Josefina Caldeira Andrade & 5 & 5 & & & & 5 \\
\hline Madre Terezinha Leoni & & 7 & & & & 7 \\
\hline Padre Bruno Pokolm & & 9 & & & & 9 \\
\hline Prof Adelina Regis - CEPAR & & 8 & 1 & 1 & 1 & 6 \\
\hline
\end{tabular}

Pelas consultas realizadas com a ferramenta, pode-se obter as seguintes informações sobre as escolas públicas estaduais de Videira/SC: $37 \%$ dos alunos consideram que pesquisas na Internet são a preferência de tarefas que os professores poderiam usar nas suas aulas; $94 \%$ dos alunos preferem aulas com o uso de tecnologias. 
A partir de consultas como estas é possível tomar algumas decisões como, por exemplo, a adoção de políticas para ofertar cursos de tecnologias para as escolas em parceria com outras instituições de ensino. Com base nos questionários aplicados os resultados mostram a grande necessidade de profissionais de informática ou de programas de capacitação dos professores, uma vez que a pesquisa revelou que muitos gostariam e precisariam melhorar o conhecimento em relação ao uso de tecnologias. Essas e outras melhorias poderiam ser realizadas por parcerias.

\section{Conclusão e trabalhos futuros}

Com a ferramenta web desenvolvida é possível realizar consultas Olap sobre os dados dos alunos e professores de escolas públicas estaduais de Videira/SC em relação ao uso/necessidade de tecnologias. A ferramenta oferece a possibilidade de analisar os dados através de uma interface amigável e que facilita a tomada de decisão por parte dos coordenadores, diretores e envolvidos na gestão em geral. Como já era esperado, os resultados da pesquisa foram muito similares aos obtidos no projeto anterior (Rosa et.al., 2016), sobre as escolas públicas municipais, principalmente em relação ao interesse dos alunos no uso de tecnologias nas aulas e o interesse dos mesmos por cursos de tecnologias.

Como sugestão de trabalhos futuros poderiam ser agrupados os dados do presente projeto e do anterior, facilitando ainda mais com a visualização integrada. A aplicação de outras técnicas de mineração dados, possivelmente sobre um questionário ampliado, também poderiam ser incrementadas possibilitando a descoberta de padrões desconhecidos na base de dados. Considerando os resultados obtidos poderia ser proposto algum curso ou parceria do Instituto com essas escolas, conforme interesse e disponibilidade de ambas as partes, uma vez que a pesquisa mostra a necessidade e o interesse de capacitação, tanto dos alunos, quanto dos professores.

\section{Referências}

KIMBALL, Ralph. Data warehouse toolkit: o guia completo para modelagem multidimensional. Rio de Janeiro: Campus, 2002.

ELMASRI, Ramez; NAVATHE, Shamkant B. Sistemas de banco de dados. São Paulo: Pearson/Addison Wesley, 2011.

MILANI, André. PostgreSQL: guia do programador. São Paulo, Novatec, 2008.

PENTAHO. Pentaho open source business intelligence. Disponível em $<$ http://www.pentaho.com>. Acesso em 20 nov. 2017.

POSTGRESQL. PostgreSQL. Disponível em <http://www.postgresql.org>. Acesso em 20 nov. 2017.

PRENSKY, Marc. Digital natives, digital immigrants - 2001. Disponível em $<$ http://www.marcprensky.com/writing/Prensky-Digital Natives,Digital ImmigrantsPart1.pdf>. Acesso em 20 nov. 2017.

ROSSI, Leila Lisiane; NACHTIGALL, Gilson Ribeiro; SENKO, Luiz Gustavo Moro; ROSA, Angela Maria Crotti da; HEINECK, Tiago; VIAN, Caroline; BOESING, Jeferson José Schneider. Software livre como apoio à tomada de decisão no ensino e uso de tecnologias: estudo de caso para as escolas públicas municipais de Videira/SC. Regae Revista de Gestão e Avaliação Educacional, Santa Maria, v. 5, n. 10, 2016, p. 95-102. 
SILBERSCHATZ, Abraham; KORTH, Henry F; SUDARCHAN, S. Sistemas de banco de dados. São Paulo: Makron Books, 2006.

WEKA, Pentaho. Open source business intelligence - data mining. Disponível em $<$ http://community.pentaho.com/projects/data-mining/>. Acesso em 20 nov. 2017.

WITTEN, Ian H; FRANK, Eibe. Data mining: practical machine learning tools and techniques. San Francisco: Elsevier, 2005.

Leila Lisiane Rossi é mestre em Ciência da Computação e professora de Informática no Instituto Federal Catarinense, campus Videira.

Endereço: Rodovia SC 135, km 125 - Bairro Campo Experimental - 89560-000 Videira - SC - Brasil.

E-mail: leila.rossi@ifc.edu.br.

Jean Carlo Corso é estudante do curso de Ciência da Computação no Instituto Federal Catarinense, campus Videira.

Endereço: Rodovia SC 135, km 125 - Bairro Campo Experimental - 89560-000 Videira - SC - Brasil.

E-mail: jeancarlocorso2@gmail.com.

Gilson Ribeiro Nachtigall é doutor em Agronomia, coordenador de pesquisa e inovação e professor nos cursos de Agronomia no Instituto Federal Catarinense, campus Videira.

Endereço: Rodovia SC 135, km 125 - Bairro Campo Experimental - 89560-000 Videira - SC - Brasil.

E-mail: gilson.nachtigall@ifc.edu.br.

Angela Maria Crotti da Rosa é especialista em Controle da Gestão Pública e coordenadora de estágios e extensão no Instituto Federal Catarinense, campus Videira.

Endereço: Rodovia SC 135, km 125 - Bairro Campo Experimental - 89560-000 Videira - SC - Brasil.

E-mail: angela.rosa@ifc.edu.br.

Luiz Gustavo Moro Senko é mestre em Ciência da Computação e professor de Informática no Instituto Federal Catarinense, campus Brusque.

Endereço: Rua Herć́lio Luz, 63 - Centro - 88350-300 - Brusque - SC - Brasil.

E-mail: luiz.senko@ifc.edu.br.

Recebido em 26 de fevereiro de 2018.

Aceito em 14 de abril de 2018. 